\title{
ITERATIVE EXTRAPOLATION ALGORITHM FOR DATA RECONSTRUCTION OVER SPHERE
}

\author{
Wen Zhang, Rodney A. Kennedy and Thushara D. Abhayapala \\ Department of Information Engineering \\ Research School of Information Sciences and Engineering \\ The Australian National University
}

Email:wen@cecs.anu.edu.au,rodney.kennedy@anu.edu.au, thushara.abhayapala@anu.edu.au

\begin{abstract}
Given limited or incomplete measurement data on a sphere, a new iterative algorithm is proposed on how to extrapolate signal over the whole sphere. The algorithm is based on a priori assumption that the Fourier decomposition of the signal on the sphere has finite degree of spherical harmonic coefficients, that is, the signal is modelimited. The algorithm is a simple iteration involving only the spherical harmonic decomposition. It is proven that the algorithm converges to the original signal over observation region and the convergence rate is lower bounded by the largest eigenvalue of an associated Fredholm integral equation.
\end{abstract}

Index Terms - Extrapolation, Spherical Harmonics, Modelimited.

\section{INTRODUCTION}

Signal extrapolation is the problem of finding an estimate of a signal outside its observation interval. Researches have proposed algorithms for bandlimited signal extrapolation over finite time interval $[1,2,3]$. Some of them have also been used on image signal processing due to the natural extension of the Fourier Transform to the $2-\mathrm{D}$ case. In this paper, the extrapolation algorithm is proposed for signal reconstruction on a sphere. The algorithm is of great practical significance to experimental data reconstruction over the whole sphere from limited or incomplete measurements, such as HeadRelated-Transfer-Function (HRTF) measurements for sound reproduction systems, the radiation field of antenna systems and earth surface data acquisition.

Let $S^{2}$ denote the unit sphere in three dimensions. It is well known that the spherical harmonics, $Y_{n}^{m}(\theta, \phi)$, for $m=-n, \cdots, n$, $n=0,1,2, \cdots$, form a complete orthogonal basis in $L^{2}\left(S^{2}\right)$ with the natural inner product [4]. Here, the angle $0^{\circ} \leq \theta \leq 180^{\circ}$ is the polar angle, or colatitude and $0^{\circ} \leq \phi \leq 360^{\circ}$ is the azimuthal angle. The expansion of a function $f \in L^{2}\left(S^{2}\right)$ can be written as

$$
f(\theta, \phi)=\sum_{n=0}^{\infty} \sum_{m=-n}^{n} F_{n m} Y_{n}^{m}(\theta, \phi) .
$$

$F_{n m}$ are the spherical harmonic coefficients of degree $n$ and order $m$ obtained by projecting $f$ onto $Y_{n}^{m}$, i.e.,

$$
F_{n m}=\int_{S^{2}} f(\theta, \phi) Y_{n}^{m}(\theta, \phi)^{*} d \Omega
$$

where $\Omega$ is the solid angle defined by $\int d \Omega=\int_{0}^{2 \pi} d \phi \int_{0}^{\pi} \sin \theta d \theta$, and the $(\cdot)^{*}$ stands for complex conjugate.
Analogous to the classical bandlimited signal extrapolation, the Extrapolation Problem solved in this paper is: find a class of functions $f(\theta, \phi)$ on a sphere from incomplete measurements $g(\theta, \phi)$ over an observation region $\left(\Gamma \subset S^{2},(\theta, \phi) \in \Gamma\right)$ based on the assumption that the function is modelimited, that is its energy is finite and its spherical harmonics coefficients $F_{n m}$ are zero above a certain degree $N$, i.e.,

$$
F_{n m}=0, \text { for }|n| \geq N .
$$

Analogous to bandlimited property of temporal signals, the assumption states that we expect the original signal $f(\theta, \phi)$ to be smooth and can be represented with a finite number of spherical harmonics. The proposed extrapolation algorithm is an iterative algorithm (extension of Papoulis algorithm [3] for bandlimited extrapolation) of reducing the mean-square error between the estimated and the original signal at successive iterations. Using this error energy reduction procedure, we will show that the algorithm converges to the original signal.

\section{ITERATIVE ALGORITHM}

The proposed method is based on an iterative algorithm involving only the spherical harmonics expansion of functions on the sphere. To determine the modelimited function $f(\theta, \phi)$ from limited measurements $g(\theta, \phi)$ over the observation region $\Gamma$, the iterative algorithm starts from computing spherical harmonic coefficients

$$
F_{n m}^{(1)}= \begin{cases}\int_{\Gamma} g(\theta, \phi) Y_{n}^{m}(\theta, \phi)^{*} d \Omega & |n|<N \\ 0 & |n| \geq N\end{cases}
$$

where $d \Omega=\sin \theta d \theta d \phi$. From (4) we can compute a modelimited function

$$
f_{1}(\theta, \phi)=\sum_{n=0}^{N-1} \sum_{m=-n}^{n} F_{n m}^{(1)} Y_{n}^{m}(\theta, \phi) .
$$

Next, we replace the segment of $f_{1}(\theta, \phi)$ over the observation region $\Gamma$ by the known data $g(\theta, \phi)$

$$
g_{1}(\theta, \phi)= \begin{cases}g(\theta, \phi) & (\theta, \phi) \in \Gamma \\ f_{1}(\theta, \phi) & \text { elsewhere. }\end{cases}
$$

Following the same procedure, at the $k^{\text {th }}$ iteration, we have

$$
F_{n m}^{(k)}= \begin{cases}\int_{S^{2}} g_{k-1}(\theta, \phi) Y_{n}^{m}(\theta, \phi)^{*} d \Omega & |n|<N \\ 0 & |n| \geq N\end{cases}
$$

and

$$
f_{k}(\theta, \phi)=\sum_{n=0}^{N-1} \sum_{m=-n}^{n} F_{n m}^{(k)} Y_{n}^{m}(\theta, \phi) .
$$


Thus,

$$
\begin{aligned}
g_{k}(\theta, \phi) & = \begin{cases}g(\theta, \phi) & (\theta, \phi) \in \Gamma \\
f_{k}(\theta, \phi) & \text { elsewhere }\end{cases} \\
& =f_{k}(\theta, \phi)+\left(D_{\Gamma}\left[f-f_{k}\right]\right)(\theta, \phi),
\end{aligned}
$$

where $D_{\Gamma}$ is defined as a space selecting operator

$$
\left(D_{\Gamma} f\right)(\theta, \phi)= \begin{cases}f(\theta, \phi) & (\theta, \phi) \in \Gamma \\ 0 & \text { elsewhere }\end{cases}
$$

Note that in the algorithm, the computed function $f_{k}(\theta, \phi)$ after each iteration is modelimited. Thus, we could form that

$$
F_{n m}^{(k)}=G_{n m}^{(k-1)} \beta_{n}^{N},
$$

where $G_{n m}^{(k-1)}$ are the spherical harmonic coefficients of $g_{k-1}(\theta, \phi)$, and $\beta_{n}^{N}$ is defined as

$$
\beta_{n}^{N}= \begin{cases}1 & |n|<N \\ 0 & |n| \geq N\end{cases}
$$

and corresponds to a modelimited operator $B_{N}$ on the sphere. That is,

$$
f_{k}(\theta, \phi)=\left(B_{N} g_{k-1}\right)(\theta, \phi) .
$$

The operator $B_{N}$ can be regarded as a low-pass spatial filtering operator, it truncates the spherical harmonics of a function to a certain degree $N$.

Therefore, this algorithm states that we start by low-pass filtering the zero extended observations. At step $k$, the low-pass filter output $f_{k}(\theta, \phi)$ is substituted by the observations $g(\theta, \phi)$ over the region $\Gamma$ and the result is low-pass filtered again to yield $f_{k+1}(\theta, \phi)$. It will be shown in the next section that $f(\theta, \phi)$ and $f_{k}(\theta, \phi)$ are in the same modelimited subspace. And it will be proven that $f_{k}(\theta, \phi)$ tend to $f(\theta, \phi)$ over measurement region $\Gamma$ as $k \rightarrow \infty$, and the convergence properties will be determined.

\section{ILLUSTRATION OF THE ALGORITHM}

Given the modelimited property of $f(\theta, \phi)$, we have

$$
f(\theta, \phi)=\left(B_{N} f\right)(\theta, \phi),
$$

and the space selected function is given by

$$
g(\theta, \phi)=\left(D_{\Gamma} f\right)(\theta, \phi) .
$$

The collection of modelimited functions forms a complete linear subspace $\mathcal{B}$ (modelimited subspace) of $L^{2}\left(S^{2}\right)$ so that all functions having the same finite degree spherical harmonic coefficients are in the same subspace. Analogously, the space selected functions form another complete linear subspace $\mathcal{D}$ (space selection subspace) of $L^{2}\left(S^{2}\right)$. Then the iterative algorithm can be regarded as an affine projection involving $D_{\Gamma}$, given in (10) and an orthogonal projection using $B_{N}$ given in (13). Thus, we have

$$
\begin{aligned}
f_{k}(\theta, \phi) & =\left(B_{N} f_{k-1}\right)(\theta, \phi)+\left(B_{N} D_{\Gamma}\left[f-f_{k-1}\right]\right)(\theta, \phi) \\
& =f_{k-1}(\theta, \phi)+\left(B_{N} D_{\Gamma}\left[f-f_{k-1}\right]\right)(\theta, \phi) .
\end{aligned}
$$

Here $f_{k}(\theta, \phi)=\left(B_{N} f_{k}\right)(\theta, \phi)$ because $f_{k}$ is in the modelimited subspace. The algorithm is in essence an iterative descent algorithm. Here by descent, we mean that in the modelimited subspace, each new point $f_{k}$ generated by the algorithm corresponds to reducing the value of some error function $\left(f-f_{k}\right)$. Intuitively, the sequence of points generated by such algorithm converges to the original function.

In characterizing the geometry of the convergence, we seek the expression for the cascade operator $B_{N} D_{\Gamma}$ on any general function $f(\theta, \phi)$ and the following eigen-decomposition is central.

Theorem 1. The cascade operator $B_{N} D_{\Gamma}$ is a self-adjoint compact Fredholm integral operator [5] on $L^{2}(\Gamma)$ defined by

$$
\left(B_{N} D_{\Gamma} f\right)(\theta, \phi)=\int_{\Gamma} K\left(\theta, \phi ; \theta_{0}, \phi_{0}\right) f\left(\theta_{0}, \phi_{0}\right) d \Omega_{0},
$$

with kernel

$$
K\left(\theta, \phi ; \theta_{0}, \phi_{0}\right)=\sum_{n=0}^{N-1} \sum_{m=-n}^{n} Y_{n}^{m}(\theta, \phi) Y_{n}^{m}\left(\theta_{0}, \phi_{0}\right)^{*} .
$$

Proof. As stated before, spherical harmonics $Y_{n}^{m}(\theta, \phi)$ are complete orthogonal basis on the sphere $S^{2}$. From (1) and (2), we have

$$
f(\theta, \phi)=\sum_{n=0}^{\infty} \sum_{m=-n}^{n}\left(\int_{S^{2}} f\left(\theta_{0}, \phi_{0}\right) Y_{n}^{m}\left(\theta_{0}, \phi_{0}\right)^{*} d \Omega_{0}\right) Y_{n}^{m}(\theta, \phi) .
$$

The above equation holds for any function $f(\theta, \phi)\left(f \in L^{2}\left(S^{2}\right)\right)$ and so holds for $\left(D_{\Gamma} f\right)(\theta, \phi) \in L^{2}(\Gamma)$, i.e.,

$$
\begin{aligned}
& \left(D_{\Gamma} f\right)(\theta, \phi) \\
& =\sum_{n=0}^{\infty} \sum_{m=-n}^{n}\left(\int_{S^{2}}\left(D_{\Gamma} f\right)\left(\theta_{0}, \phi_{0}\right) Y_{n}^{m}\left(\theta_{0}, \phi_{0}\right)^{*} d \Omega_{0}\right) Y_{n}^{m}(\theta, \phi) \\
& =\sum_{n=0}^{\infty} \sum_{m=-n}^{n}\left(\int_{\Gamma} f\left(\theta_{0}, \phi_{0}\right) Y_{n}^{m}\left(\theta_{0}, \phi_{0}\right)^{*} d \Omega_{0}\right) Y_{n}^{m}(\theta, \phi) .
\end{aligned}
$$

The modelimited operator $B_{N}$ truncates the spherical harmonics to a certain degree $N$; and we have

$$
\begin{aligned}
& \left(B_{N} D_{\Gamma} f\right)(\theta, \phi)= \\
& \sum_{n=0}^{N-1} \sum_{m=-n}^{n}\left(\int_{\Gamma} f\left(\theta_{0}, \phi_{0}\right) Y_{n}^{m}\left(\theta_{0}, \phi_{0}\right)^{*} d \Omega_{0}\right) Y_{n}^{m}(\theta, \phi) .
\end{aligned}
$$

This is equivalent to (17) and (18). Since the kernel satisfies

$$
K\left(\theta, \phi ; \theta_{0}, \phi_{0}\right)=K\left(\theta_{0}, \phi_{0} ; \theta, \phi\right)^{*},
$$

the operator in (17) is self-adjoint. Further the operator is compact and positive.

Over the observation region $(\theta, \phi) \in \Gamma,(17)$ has a set of real positive eigenvalues $\lambda_{i}\left(\lambda_{i}<1\right)^{1}$ and orthogonal eigenvectors $\varphi_{i}(\theta, \phi)$ (corresponding to distinct eigenvalues). That is,

$$
\begin{aligned}
& \int_{\Gamma} \varphi_{i}\left(\theta_{0}, \phi_{0}\right) \sum_{n=0}^{N-1} \sum_{m=-n}^{n} Y_{n}^{m}(\theta, \phi) Y_{n}^{m}\left(\theta_{0}, \phi_{0}\right)^{*} d \Omega_{0} \\
& =\lambda_{i} \varphi_{i}(\theta, \phi),
\end{aligned}
$$

where

$$
\int_{\Gamma} \varphi_{i}(\theta, \phi) \varphi_{j}(\theta, \phi)^{*} d \Omega=\delta_{i j}
$$

\footnotetext{
${ }^{1}$ Note the function total energy is reduced after the $B_{N} D$ operation; and eigenvalues show the fractional function energy passed under such a transform.
} 
Notation used in (23) reveals that eigenvectors and eigenvalues are functions of the observation region $\Gamma$, and the given value of the spherical harmonic coefficients degree $N$. Fig. 1 gives an example of the first two dominant eigenvectors for $\Gamma=(0 \leq \theta \leq$ $120^{\circ}, 0 \leq \phi \leq 200^{\circ}$ ) with $N=4$. And as we see from Fig. $2, \lambda_{0}$ (the largest eigenvalue corresponds to the eigenfunction with smallest energy loss) increases with the area of observation region $\Gamma$ when the value of $N$ is fixed. In the next section, we will use the derived orthogonal eigenvectors to prove the convergence of the algorithm.

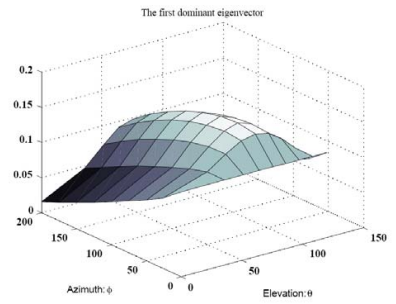

(a) $\varphi_{0}(\theta, \phi)$

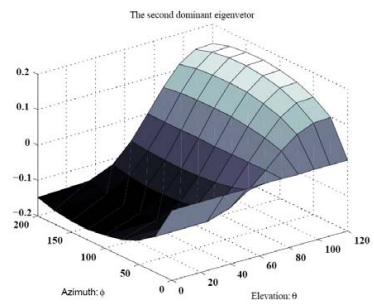

(b) $\varphi_{1}(\theta, \phi)$
Fig. 1. An example of the dominant two eigenvectors for observation region $\Gamma=\left(0 \leq \theta \leq 120^{\circ}, 0 \leq \phi \leq 200^{\circ}\right)$ and spherical harmonic coefficients degree $N=4$.

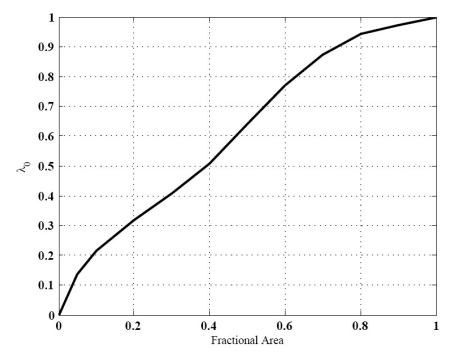

Fig. 2. An example of $\lambda_{0}$ increasing with the observation region, where the colatitude observations increase from $0^{\circ}$ to $180^{\circ}$ and the full range of azimuthal observation is assumed. The observation region is represented by fractional area $\left(\int_{\Gamma} d \Omega / 4 \pi\right)$, the solid angles in fractions of the sphere.

\section{CONVERGENCE}

To prove the convergence of the iteration, by the Spectral Theorem for self-adjoint compact operators [6], we use $\varphi_{i}(\theta, \phi)$ as orthogonal basis to expand the function $f(\theta, \phi)$ over the region $\Gamma$

$$
f(\theta, \phi)=\sum_{i=0}^{\infty} \alpha_{i} \varphi_{i}(\theta, \phi),(\theta, \phi) \in \Gamma .
$$

Similar results as in [3] are obtained.

Theorem 2. The $k^{\text {th }}$ iteration of the algorithm is given by

$$
f_{k}(\theta, \phi)=\sum_{i=0}^{\infty} \alpha_{i}\left[1-\left(1-\lambda_{i}\right)^{k}\right] \varphi_{i}(\theta, \phi),(\theta, \phi) \in \Gamma
$$

where $\lambda_{i}$ and $\varphi_{i}$ are introduced after Theorem 1, and $\alpha_{i}$ are the function expansion coefficients.
Proof. At the first step

$$
f_{1}(\theta, \phi)=\left(B_{N} D_{\Gamma} f\right)(\theta, \phi)=\sum_{i=0}^{\infty} \alpha_{i} \lambda_{i} \varphi_{i}(\theta, \phi),(\theta, \phi) \in \Gamma .
$$

Suppose at the $k^{\text {th }}$ step

$$
f_{k}(\theta, \phi)=\sum_{i=0}^{\infty} \alpha_{i} A_{k}^{(i)} \varphi_{i}(\theta, \phi),(\theta, \phi) \in \Gamma .
$$

From (16)

$$
\begin{aligned}
f_{k}(\theta, \phi) & =f_{k-1}(\theta, \phi)+\left(B_{N} D_{\Gamma}\left[f-f_{k-1}\right]\right)(\theta, \phi) \\
& =\sum_{i=0}^{\infty} \alpha_{i}\left(A_{k-1}^{(i)}+\left(1-A_{k-1}^{(i)}\right) \lambda_{i}\right) \varphi_{i}(\theta, \phi),(\theta, \phi) \in \Gamma .
\end{aligned}
$$

Thus

$$
A_{k}^{(i)}=A_{k-1}^{(i)}+\left(1-A_{k-1}^{(i)}\right) \lambda_{i} .
$$

Solving the recursion with the initial condition $A_{1}^{(i)}=\lambda_{i}$, we conclude that

$$
f_{k}(\theta, \phi)=\sum_{i=0}^{\infty} \alpha_{i}\left[1-\left(1-\lambda_{i}\right)^{k}\right] \varphi_{i}(\theta, \phi),(\theta, \phi) \in \Gamma .
$$

Theorem 3. Over measurement region $\Gamma$,

$$
f_{k}(\theta, \phi) \rightarrow f(\theta, \phi) \text {, as } k \rightarrow \infty .
$$

Proof. From (25) and Theorem 2, the error

$$
e_{k}(\theta, \phi)=f(\theta, \phi)-f_{k}(\theta, \phi)
$$

is given by

$$
e_{k}(\theta, \phi)=\sum_{i=0}^{\infty} \alpha_{i}\left(1-\lambda_{i}\right)^{k} \varphi_{i}(\theta, \phi),(\theta, \phi) \in \Gamma .
$$

The mean-square value $E_{k}$ is written as

$$
E_{k}=\int_{\Gamma} e_{k}(\theta, \phi)^{2} d \Omega=\sum_{i=0}^{\infty} \alpha_{i}^{2}\left(1-\lambda_{i}\right)^{2 k}
$$

As signal energy $E=\sum_{i=1}^{\infty}\left|\alpha_{i}\right|^{2}$ is finite; hence, there is an integer $P$ that

$$
\sum_{i>P}\left|\alpha_{i}\right|^{2}<\varepsilon_{1}
$$

where $\varepsilon_{1}$ is an arbitrary small value and $\varepsilon_{1}>0$. Eigenvalues $0<$ $\lambda_{i}<1$ and tend to zero as $i \rightarrow \infty$, we have $1-\lambda_{i}<1$ and

$$
\left(1-\lambda_{i}\right) \leq\left(1-\lambda_{P}\right), \text { for } i \leq P .
$$

Then

$$
\begin{aligned}
E_{k} & =\sum_{i=0}^{P}\left|\alpha_{i}\right|^{2}\left(1-\lambda_{i}\right)^{2 k}+\sum_{i>P}^{\infty}\left|\alpha_{i}\right|^{2}\left(1-\lambda_{i}\right)^{2 k} \\
& <\left(1-\lambda_{P}\right)^{2 k} \sum_{i=0}^{P}\left|\alpha_{i}\right|^{2}+\sum_{i>P}^{\infty}\left|\alpha_{i}\right|^{2}<\left(1-\lambda_{P}\right)^{2 k} E+\varepsilon_{1} .
\end{aligned}
$$


As $k \rightarrow \infty,\left(1-\lambda_{P}\right)^{2 k} \rightarrow 0$. Thus $E_{k}$ is always less than an arbitrary small value $\varepsilon=\varepsilon_{1}+\varepsilon_{2}$ where $\left(1-\lambda_{P}\right)^{2 k} E<\varepsilon_{2}$. The mean-square error value between the estimated and original signal at successive iterations is reduced, that is, $e_{k}(\theta, \phi) \rightarrow 0$ in the mean as $k \rightarrow \infty$. The estimates $f_{k}$ and original function $f$ are in the same modelimited subspace; thus Theorem 3 actually means the estimated signal tending to the original function over the whole sphere .

Notation used in (34) reveals that the estimation error is a function of eigenvalues of the integral equation (23). Considering the eigenvalue properties stated in section 3 , two remarks about the iterative algorithm are:

1. The convergence of the iteration algorithm is lower bounded by the largest eigenvalue $\lambda_{0}$, as from (34), the minimum estimation error after each iteration is $\sum_{i=0}^{\infty} \alpha_{i}\left(1-\lambda_{0}\right)^{k} \varphi_{i}(\theta, \phi)$.

2. As shown in Fig. 2, $\lambda_{0}$ increases with the area of observation region $\Gamma$, which means that measurements of larger observation region have faster initial convergence and thus would tend to require a smaller number of iterations.

\section{NUMERICAL SIMULATION RESULTS}

In this section, we apply the proposed method to extrapolate incomplete data on a sphere and examine the algorithm performance. The data is artificially generated by assigning specific spherical harmonic coefficients to degree $N$ and then computed from functional spherical harmonics decomposition formula (1). In the following example, the signal is assumed to be mode limited to $N=4$ and random values are assigned to the spherical harmonic coefficients. The observations are given for $0 \leq \theta \leq 140^{\circ}, 0 \leq \phi \leq 300^{\circ}$. Fig. 3 shows the original signal, the given observations and the estimated signal after 30 iterations. It can be seen that the algorithm provides very accurate extrapolation results.

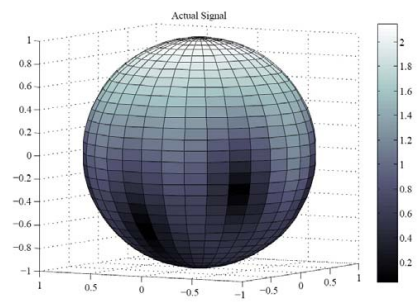

(a) Original signal $f(\theta, \phi)$.

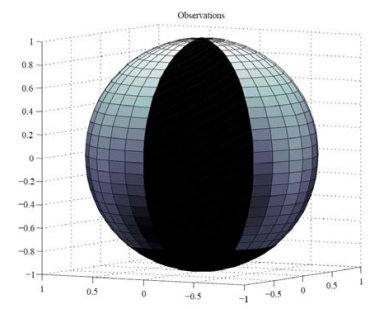

(b) Given Observations $g(\theta, \phi)$

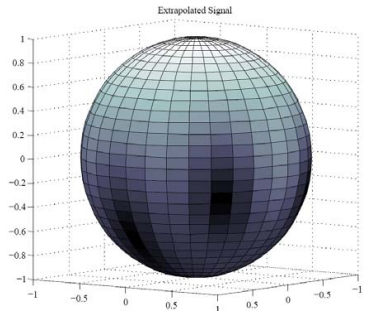

(c) Extrapolated signal $f_{k}(\theta, \phi)$
Fig. 3. An example of data reconstruction on the sphere using the proposed iterative algorithm. The observations in (b) are given for $0 \leq \theta \leq 140^{\circ}, 0 \leq \phi \leq 300^{\circ}$ with $N=4$. Extrapolation results in (c) are the estimates after 30 iterations. The color scale shows the signal magnitude; and the pure black means no observation made.

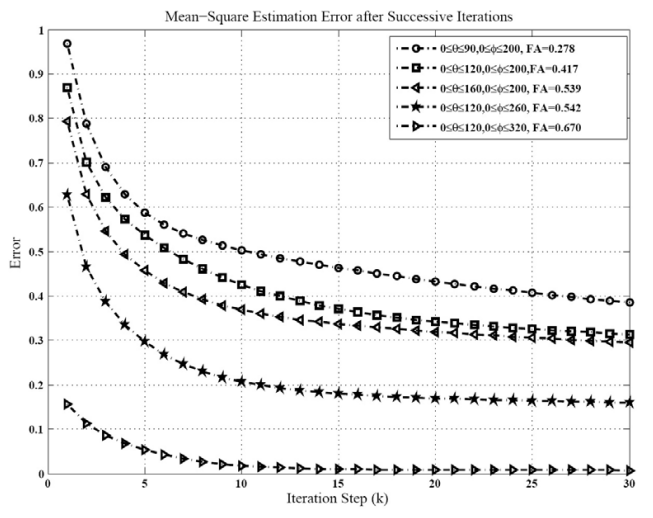

Fig. 4. Mean square error between the estimated and original signal for different data observation regions. FA stands for fractional area of the observation region.

The mean-square estimation error between the estimated and original signal after each iteration for different observation regions are shown in Fig. 4. Several observations are: firstly, the estimation error is exponentially decaying with the iteration step, demonstrating that the convergence of $F_{n m}^{k}$ to the unknown modelimited spherical harmonic coefficients $F_{n m}$ is remarkably rapid. The smaller error and faster convergence associated with the measurements of larger observation region support the remarks of the algorithm in Section 4.

\section{CONCLUSION}

This paper has presented an iterative algorithm for signal extrapolation over the whole sphere from limited or incomplete measurements based on the priori knowledge that the signal spherical harmonic expansion is finite dimensional. The algorithm can be regarded as projections involving two subspaces, the modelimited subspace and the space selection subspace, corresponding to the modelimited signal and given measurements. The iterative algorithm reduces the meansquare error between the estimated and original signal at successive iterations. Numerical simulation results demonstrate the fast convergence of the algorithm to the original function.

\section{REFERENCES}

[1] R.B. Blackman and J.W. Tukey, The Measurement of Power Spectra, New York: Dover, 1958.

[2] D.S. Slepian, H.J. Landau, and H.O. Pollak, "Prolate spheroidal wave functions, Fourier analysis and uncertainty principle I, II," Bell Syst. Tech. Jounal, vol. 40, no. 1, pp. 43-84, 1961.

[3] A. Papoulis, "A new algorithm in spectral analysis and bandlimited extrapolation," IEEE Trans. Circuits Syst., vol. CAS-22, pp. 735-742, Sept. 1975.

[4] D. Colton and R. Kress, Inverse Acoustic and Electromagnetic Scattering Theory, 2nd Edition, Springer-Verlag, 1998.

[5] A.G. Ramm, "A simple proof of the Fredholm alternative and a characterization of the Fredholm operators," American Mathematical Monthly, vol. 108, pp. 855-860, 2001.

[6] L. Debnath and P. Mikusinski, Introduction to Hilbert Spaces with Applications, 3rd Edition, Academic Press, 2005. 